\title{
EVALUATION OF VARIOUS ADAPTIVE VOLTAGE POSITIONING (AVP) SCHEMES FOR COMPUTER POWER SOURCES
}

\author{
Dan Chen*, Martin Lee, and Ching-Jan Chen
}

\begin{abstract}
The latest computer CPUs require the use of DC power converters with AVP. Three commonly-used AVP control schemes were compared using small-signal loop gain analysis. From the comparison results emerges the fourth scheme proposed recently: the Native current-mode AVP scheme (NAVP). NAVP provides inherent phase current balancing, cycle-to-cycle protection, large stability margin, good line regulation, and constant output impedance. It compares favorably against the other three schemes.
\end{abstract}

Key Words: DC converters, AVP, adaptive voltage positioning, output impedance, NAVP.

\section{INTRODUCTION}

In recent years, the electrical sources for powering the latest computer central processors (CPUs) have received tremendous attention. As the performances of CPUs mandate high power efficiency, small capacitor size, and good transient behavior of the DC converters, much endeavor has been directed to address the issues. One of the recent trends to meet the requirements, the so-called adaptive voltage position (AVP) scheme, has been a hot research topic recently (Zhang, 2000). AVP specifically shapes the converter output load line to reduce overall CPU power dissipation and reduce the converter output capacitor sizes. To meet such a load line requirement and converter transient specification, constant converter output impedance is desirable (Yao et al., 2002a). Therefore, one of the tasks in AVP feedback circuit design is to shape constant output impedance of a specific value mandated by CPU manufacturers.

Because of this new requirement, some of the conventional design guidelines for power converters must be changed. For example, larger control loop gain in general improves the converter performance such as line regulation, output impedances and transient responses. In general, this design philosophy

*Correspording author. (Tel: 886-2-33663542; Email: chend@cc.ee.ntu.edu.tw)

The authors are with the Department of Electrical Engineering, National Taiwan University, Taipei, Taiwan 106, R.O.C. applies to both current-mode and voltage-mode control schemes. Normally, the top priority of the loop gain compensation design is to push the loop gain as high as possible as long as the feedback is stable. However, for AVP supplies, the priority of the feedback compensator design has been altered. In order to meet the transient response and load line requirements, the output impedance must be shaped properly by the compensator design (Yao et al., 2002b).

In this paper, four AVP control schemes will be evaluated and compared. Loop gain analysis will be used to investigate the characteristics. Two-loopgain-functions $\left(T_{1}\right.$ and $\left.T_{2}\right)$ approach was used because it provides insight into the AVP designs and performance.

\section{CONTROL BLOCK DIAGRAMS OF VARIOUS CONTROL SCHEMES}

Figure 1 shows three commonly-used control schemes to achieve AVP. Fig. 1(a) uses conventional peak current mode control in which the output voltage is sensed and fed back through a compensator to determine, in conjunction with the inductor peak current and the external ramp, the duty cycle. Fig. 1(b) shows the AVP- scheme in which the sensed inductor current information is summed with the output voltage and fed back through the inverting (negative) terminal of a compensator to determine, in conjunction with an external saw-tooth ramp, the duty cycle. For 


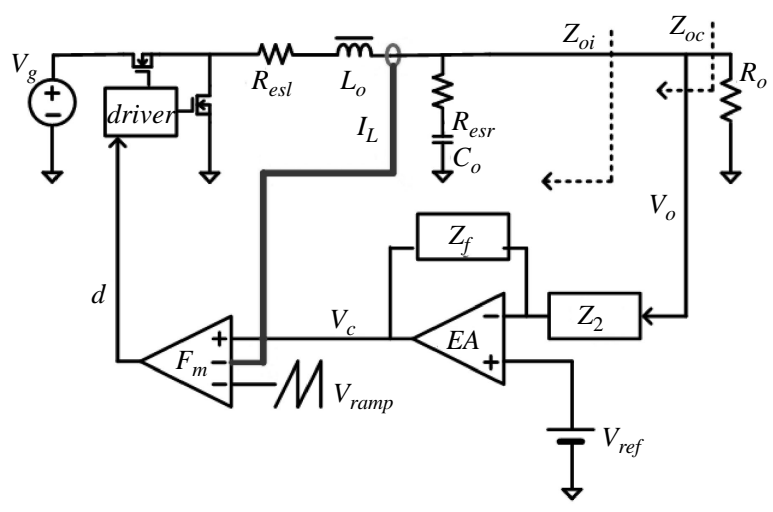

(a) Current-mode control

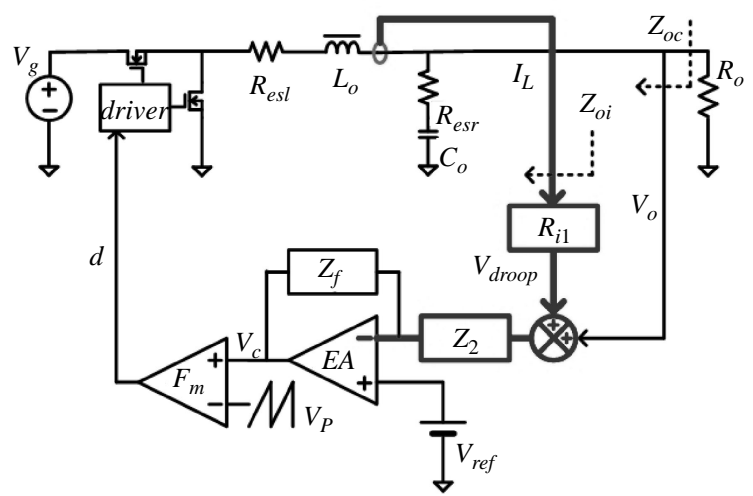

(b) AVP- control

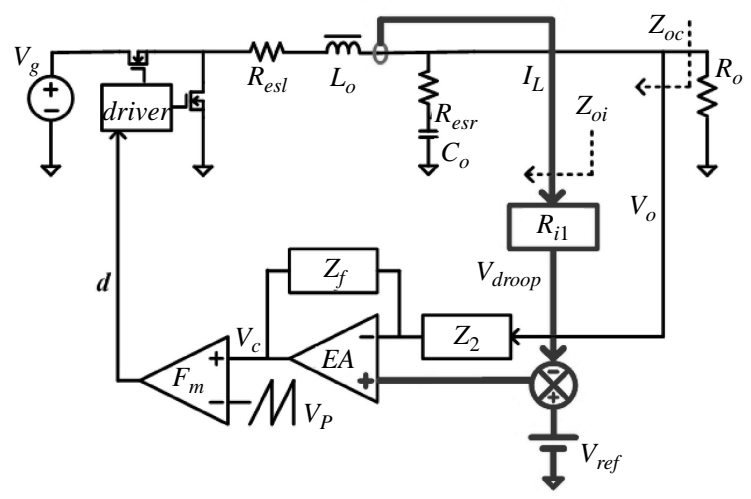

(c) AVP+ control

Fig. 1 Circuit diagrams of three commonly-used AVP schemes

that reason, it's called AVP- in this paper. Fig. 1(c) shows the AVP+ scheme in which the sensed inductor current information is fed back to sum with the reference voltage to effectively adjust the reference voltage according to the load condition and the output voltage is fed back through the compensator. Because the sensed current information is added to the positive terminal of the compensator, it's called AVP+ in this paper. The control block diagrams of the three schemes are shown in Fig. 2, and Table 1 shows the transfer functions of the various blocks. (Lee et al., 2006)

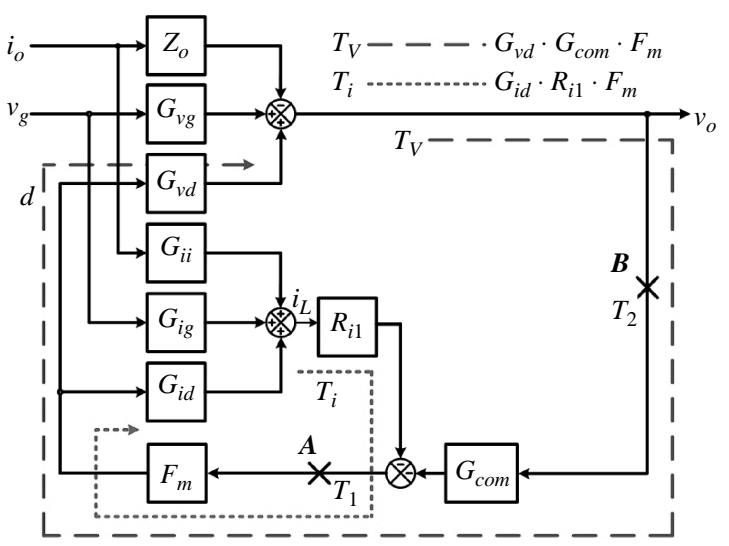

(a) Current-mode control

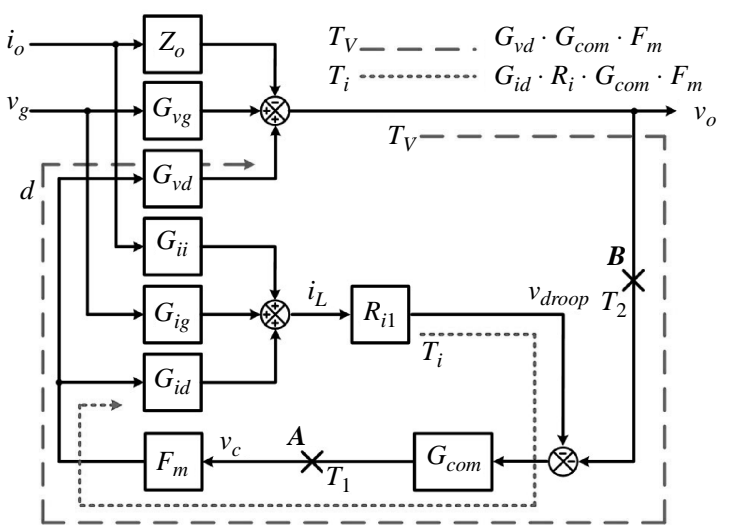

(b) AVP- control

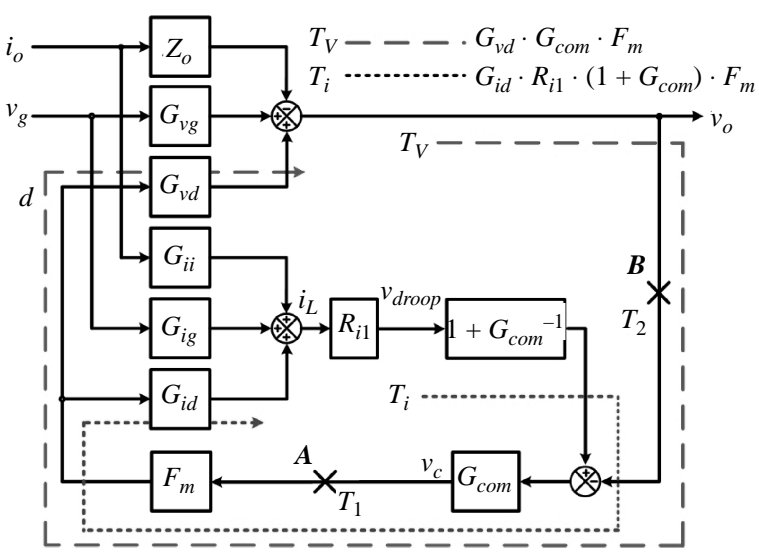

(c) AVP+ control

Fig. 2 Small-signal block diagrams of the three control schemes

\section{INVESTIGATING THE CONTROL CHARACTERISTICS}

Because there are two loops in the control block diagrams, there are, two different loop gain transfer functions. Depending on where the loop gain is measured, as shown by Fig. 2, there are two loop gain functions $T_{1}$ and $T_{2}$. In the past, there have been two approaches to investigate the control behaviors when 
Table 1 Small-signal transfer functions

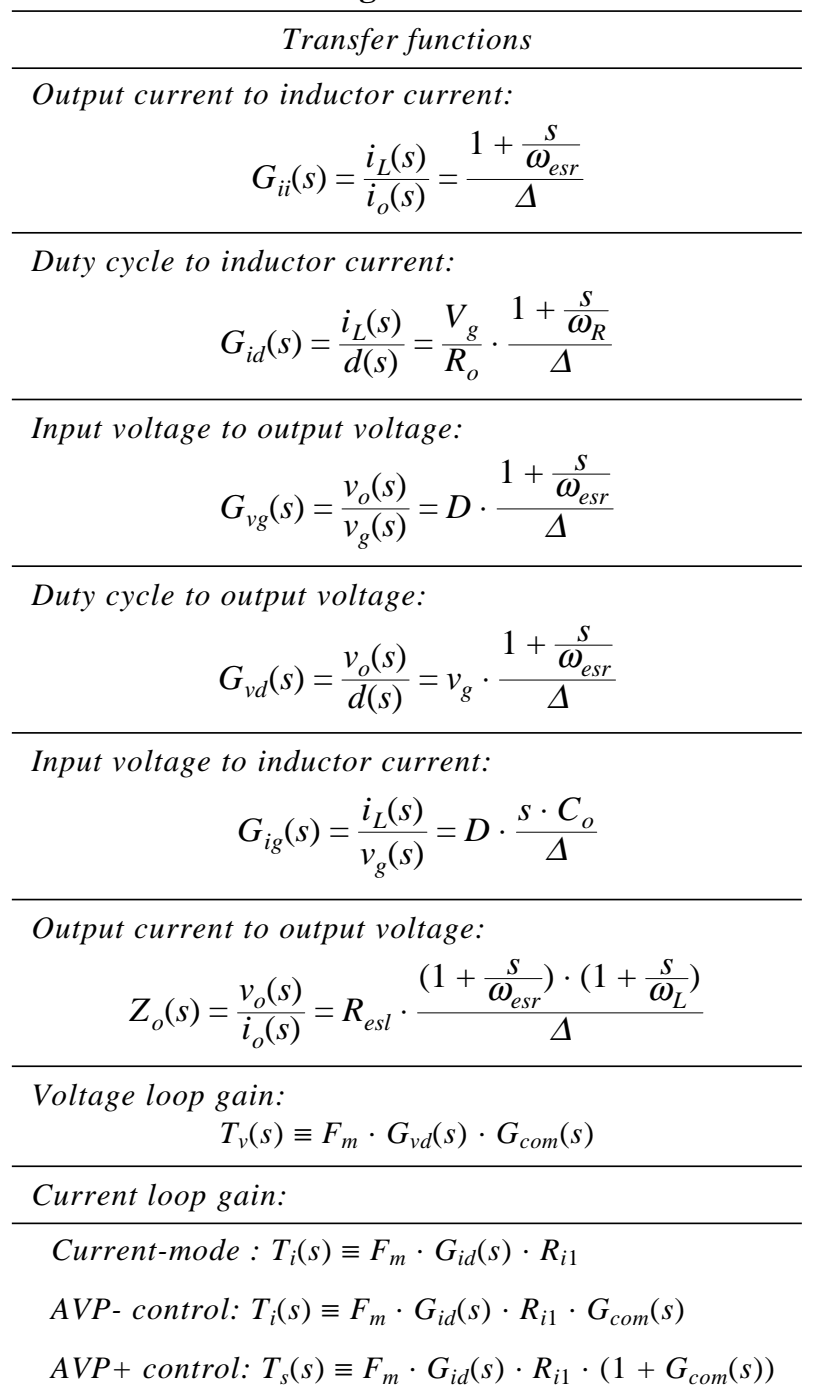

Compensator gain:

$$
G_{c o m}(s)=\frac{-z_{f}(s)}{z_{2}(s)}
$$

Modulator gain:

$$
F_{m}=\frac{d(s)}{v_{c}(s)}=\frac{1}{V_{P}}
$$

\begin{tabular}{ll}
\hline \multicolumn{1}{c}{ Parameters } \\
$\omega_{o}=\sqrt{\frac{R_{o}}{L_{o} \cdot C_{o} \cdot\left(R_{o}+C_{o}\right)}}$ & $\omega_{R}=\frac{1}{R_{o} \cdot C_{o}}$ \\
$\omega_{e s r}=\frac{1}{R_{e s r} \cdot C_{o}}$ & $\omega_{L}=\frac{R_{e s l}}{L_{o}}$ \\
$Q=\frac{1}{\omega_{o}} \cdot \frac{1}{R_{e s r} \cdot C_{o}+\frac{L_{o}}{R_{o}}}$ & $\Delta=1+\cdot \frac{s}{Q \cdot \omega_{o}}+\frac{s^{2}}{\omega_{o}}$ \\
\hline
\end{tabular}

a two-loop current-mode converter was analyzed.

One was to use loop gain functions $T_{1}$ and $T_{2}$ to investigate the control characteristics and to design the compensators for current-mode DC/DC converter (Ridley et al., 1988). Because of mathematical complexity, this approach was later replaced, however, by a more elegant and easier-to-use continuous-time model (Ridley, 1991). But in this paper, the former approach was used to investigate the intricate relationships in the AVP control characteristics because in authors' view, it provides better insight into the AVP control which requires a constant output impedance converter design.

\section{1. $T_{1}$ and $T_{2}$}

As shown in Fig. 2, loop gains $T_{1}$ and $T_{2}$ are obtained at different points of the systems. Eqs.(1) and (2) show the functional relationships of the two loop gain functions, $T_{i}$ and $T_{v}$ (Ridley et al., 1988). Both $T_{i}$ and $T_{v}$ are functions of power-stage and feedback parameters shown in Table 1

$$
\begin{aligned}
& T_{1}(s)=T_{i}(s)+T_{v}(s) \\
& T_{2}(s)=\frac{T_{v}(s)}{1+T_{i}(s)} .
\end{aligned}
$$

\section{Output Impedance and Loop Gain Functions}

As mentioned, the top priority of the feedback design to accomplish AVP is to achieve constant converter output impedance under the closed-loop condition. Eq. (3) shows the output impedance with current-loop closed but voltage loop open, $Z_{o i}$, as a function of power-stage and feedback parameters. Eq. (4) shows the output impedance $Z_{o c}$ when both loops are closed. $T_{2}$ plays a major role in shaping $Z_{o c}$ as can be seen from Eq. (4)

$$
\begin{aligned}
& Z_{o i}(s)=\frac{Z_{o}(s)\left(1+T_{i}(s)\right)+F_{m} \cdot G_{i i}(s) \cdot G_{v d}(s) \cdot R_{i 1}}{1+T_{i}(s)} \\
& Z_{o c}(s)=\frac{Z_{o i}(s)}{1+T_{2}(s)} .
\end{aligned}
$$

\section{Line Regulations and Loop Gain Functions}

Closed-loop line regulation, or audio-susceptibility Gvgc, however, is closely related to $T_{1}$ as Eq. (5) shows.

$$
G_{v g c}(s)=\frac{G_{v g}(s)}{1+T_{1}} .
$$

Definitions of all the symbols used in these equations can be found in Table 1 . 


\section{FEEDBACK DESIGN FOR AVP}

The five equations given in the last section and the various transfer functions given in Table 1 will be used in this section to investigate the feedback design to achieve AVP and other converter measures. It's important to point out that $T_{1}$ and $T_{2}$ are closely related to converter closed-loop performances. Therefore, $T_{1}$ and $T_{2}$ must be properly shaped to accomplish the design goals. However, $T_{1}$ and $T_{2}$ are not directly related to compensator design. They must be related through the two functions $T_{i}$ and $T_{v}$ to the compensator design. As can be seen from Table 1, $T_{i}$ and $T_{v}$ are, in general, influenced by the compensator gain $G_{c o m}$. Therefore, the designers can shape $G_{\text {com }}$ such that it affects $T_{i}$ and $T_{v}$ functions which indirectly affect $T_{1}$ and $T_{2}$ to accomplish the design goals. Complexity is the main reason, in authors' belief, why the $T_{1}$-and- $T_{2}$ approach is less popular. But in the case of AVP design, it's authors' belief that this approach provides much better insight into the intricate relationships and requirements.

The top consideration of the feedback design to accomplish AVP is to design for constant converter output impedance. The system must be stable, of course. As results of these requirements, other performances may not be totally at designers' discretion.

\section{1. $T_{i}$ and $T_{v}$ Dependency on $G_{c o m}$}

It's important to point out that $T_{v}$ depends on $G_{c o m}$ in all three control schemes. $T_{i}$ is dependent on $G_{c o m}$ except in the current-mode scheme. These relationships are shown in Table 1. One of the feedback design tasks is to choose proper $G_{\text {com }}$ so that converter performance goals can be met. The subtle differences of these dependencies lead to somewhat different performance behaviors among the three schemes.

\section{Desirable $\boldsymbol{T}_{2}$ to Achieve Constant Output Imped- ances}

Figure 3 shows the converter output-impedance under various feedback conditions. $Z_{o}$ is open-loop output impedance. $Z_{o i}$ is that when only the current loop is closed. As can be seen from the figure, the magnitude of output impedance increases when the current loop is closed. And $Z_{o c}$ is the desirable output impedance when both voltage and current loops are closed.

In order to achieve the desirable constant $Z_{o c}$, the loop gain $T_{2}$ must be approximately equal to the shaded area in Fig. 3, i.e., $T_{2}$ low-frequency gain must be $R_{o} / R_{e s r}$ and its bandwidth is $\omega_{e s r}$. This conclusion is obtained through the reasoning of Eq. (4) under the assumption that $T_{2}>1$ at low frequency, which is normally valid. (Lee et al., 2006)

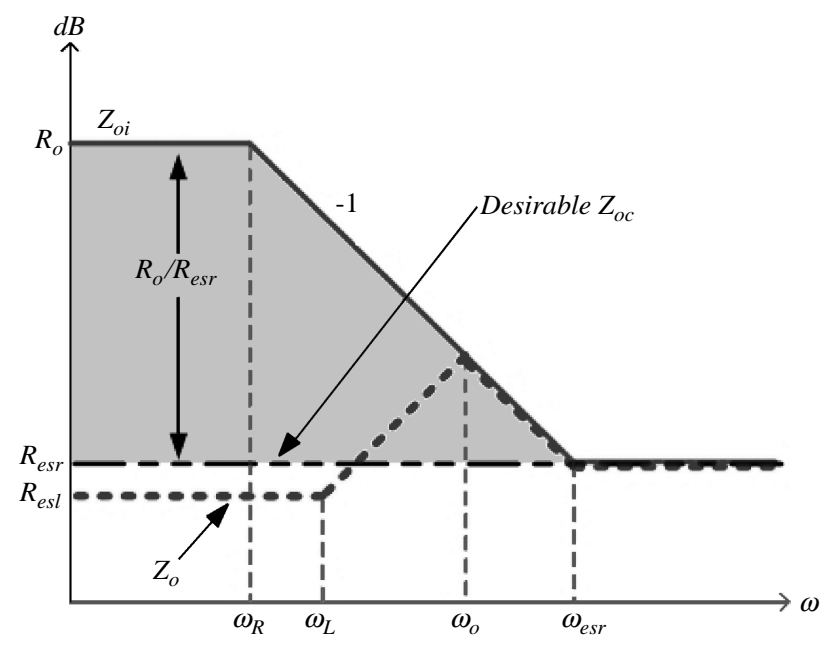

Fig. 3 Buck converter output impedances under different control conditions

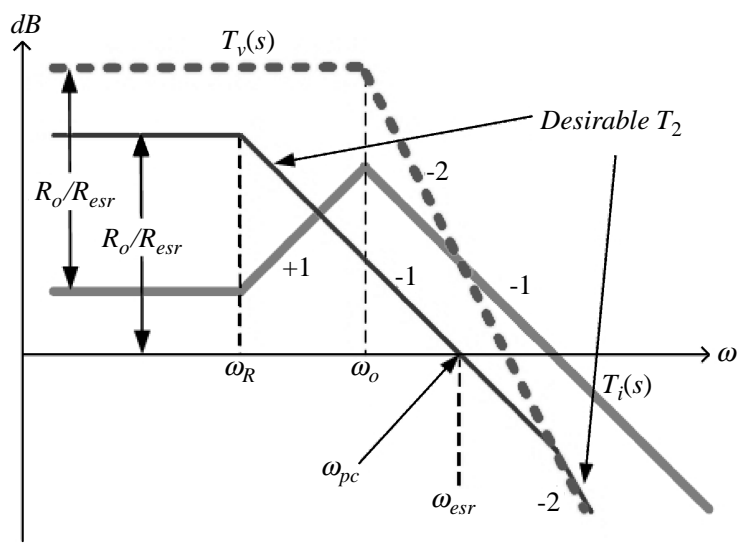

Fig. 4 Plots of proper $|T v|$ to achieve constant output impedance for the current-mode control schemes

\section{How to Shape Desirable $T_{2}$ by $G_{c o m}$ ?}

From Table 1, $T_{i}$ for current-mode scheme is the product of three factors $F_{m}, G_{i d}$ and $R_{i l} . \quad F m$ and Ril are both constant. Therefore, $\left|T_{i}\right|$ frequency response curve is similar to $G_{i d}$ 's, with two poles and one zero as shown in Fig. 4. To achieve the desirable $\left|T_{2}\right|$ in a current-mode scheme under a given $\left|T_{i}\right|$ curve, $\left|T_{v}\right|$ must be as shown. This is because $\left|T_{2}\right|$ is approximately the same as $T_{v} / T_{i}$ if $\left|T_{i}\right| \gg 1$. To obtain such a $T_{v}$, $\left|G_{\text {com }}\right|$ must be shaped as shown in Fig. 5: a compensation pole at $\omega_{e s r}$ and magnitude of $\left|T v /\left(F m \cdot G_{v d}\right)\right|$ at low frequency.

For the other two schemes, the situation is somewhat different. For AVP-, because both $T i$ and $T_{v}$ contain the same factor $G_{c o m}, T_{2}$ low-freq behavior is independent of $G_{\text {com }}$ as long as $\left|T_{i}\right| \gg>1$. For $\mathrm{AVP}+$, the conclusion is the same if both $\left|T_{i}\right| \gg 1$ and $\left|G_{\text {com }}\right| \gg 1$. Therefore, a low-freq compensating pole and a zero slightly less than $\omega_{o}$ can be placed in 


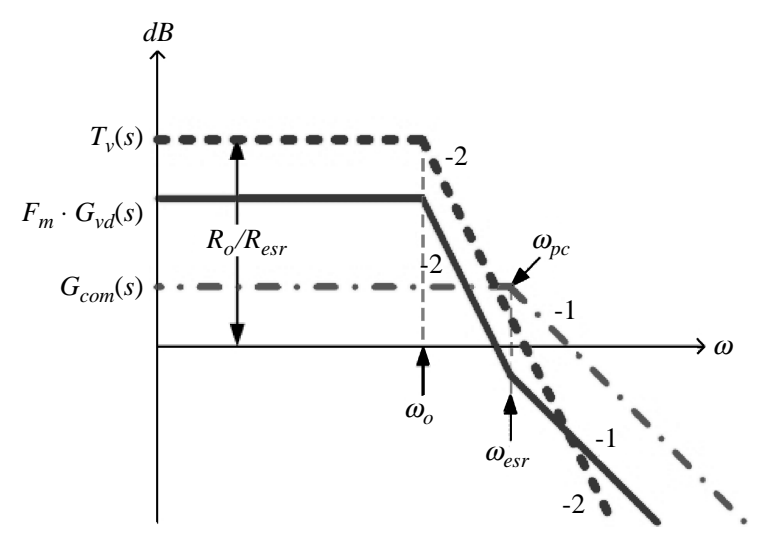

Fig. 5 Proper Gcom to shape $T v$ such that desirable $T 2$ can be obtained for current-mode scheme

AVP+ and AVP- scheme compensation without altering the $T_{2}$ low-frequency behavior to achieve desirable $T_{2}$ to achieve constant output impedance. This compensation pole, however, raises low frequency gain of $\left|T_{1}\right|$ and therefore contributes to converter line regulation. This is to be discussed later.

\section{Dependency of $T_{2}$ Stability Margin on $G_{c o m}$}

From Eq. 2, the larger the $T_{i}$ phase margin, the less peaking the $\left|T_{2}\right|$ plot exhibits and the larger the $T_{2}$ stability margin. $G_{c o m}$ has no effect on $T_{i}$ and therefore no effect on the current-mode $T_{2}$ stability margin. For the other two schemes, however, $G_{\text {com }}$ affects $T_{i}$ and therefore the $T_{2}$ stability margin. A comparison was made under comparable conditions about the margins (Lee et al., 2006). Fig. 6 shows the margin for all three cases. The current-mode scheme is the best and the AVP-scheme is the worst.

\section{Zoc Dependency on $G_{\text {com }}$}

$Z_{o c}$ is essentially a constant except near $\left|T_{2}\right|$ crossover frequency $\omega_{e s r}$ where there is a peaking for all three cases. $T_{2}$ phase margin directly affects $Z_{o c}$ peaking phenomenon near the ESR frequency. The smaller the stability margin, the larger the $\left|Z_{o c}\right|$ peaking. Fig. 7 shows the comparison. Not surprisingly, it favors the current-mode scheme.

\section{Line regulation Dependency on $G_{c o m}$}

Because utmost concern is given to $\left|Z_{o c}\right|, T_{2}$ must be shaped properly as described above. However, this inevitably sets low-freq. gain of $T_{i}$ and $T_{v}$ relatively low in the case of the current-mode AVP. According to Eq. 1, this means $T_{1}$ 's low-freq. gain is relatively low compared with the other two schemes compensated by a low-freq. pole as mentioned in an early

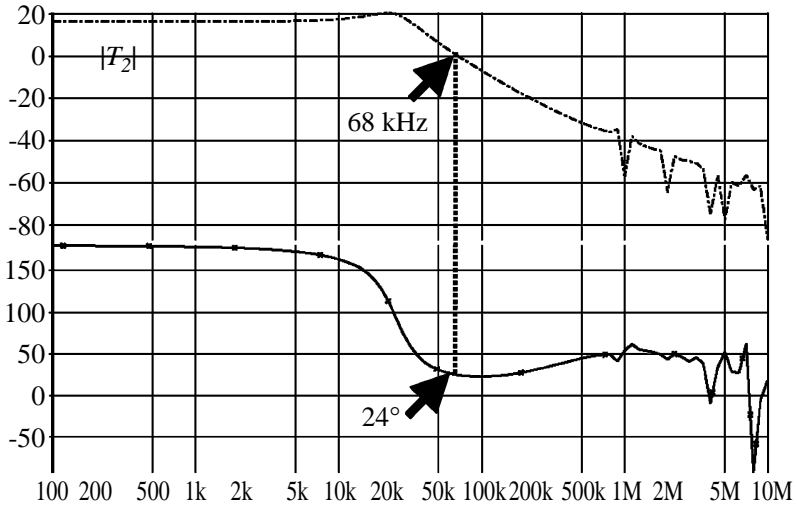

freq / Hertz

(a) current-mode control

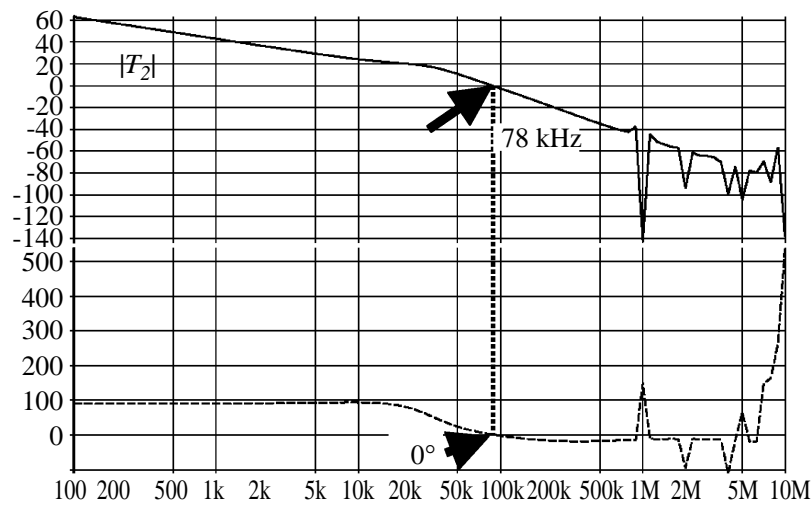

freq / Hertz

(b) AVP- control

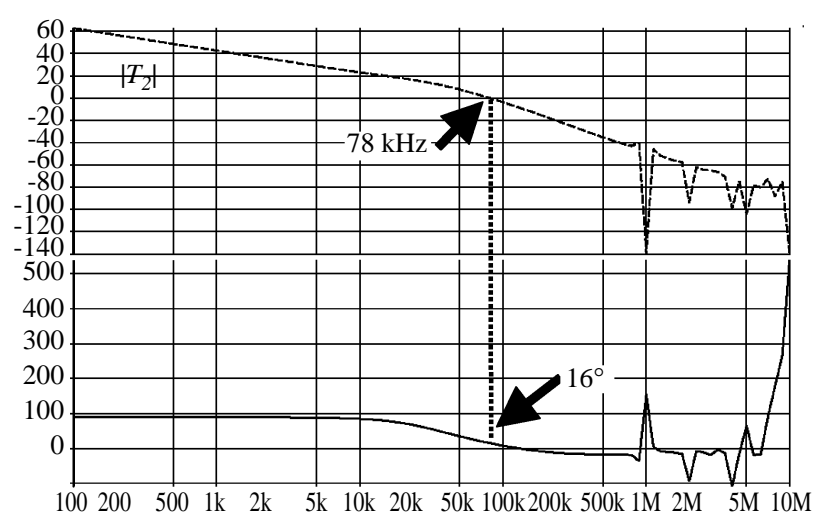
freq / Hertz

(c) AVP+ control

Fig. 6 The utter loop gains, $T_{2}$ and phase margin

subsection entitled "How to shape desirable $T_{2}$ by $G_{\text {com }}$ ?" This explains why the line regulation of current-mode AVP is relatively poor. Fig. 8 shows the comparison of the line regulation performances of the three schemes.

\section{PEAK CURRENT MODE WITH NATIVE AVP}

From the discussion in the last section, it's 


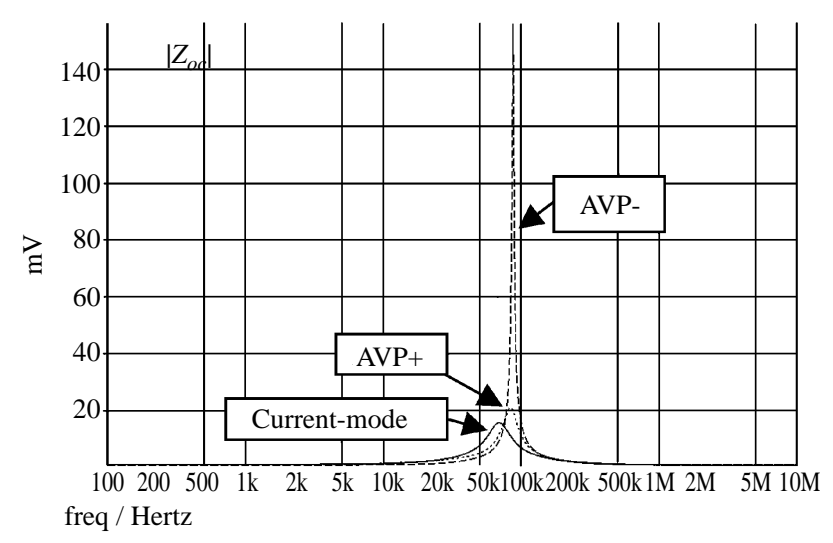

Fig. 7 The closed loop output impedance, $\left|Z_{o c}\right|$

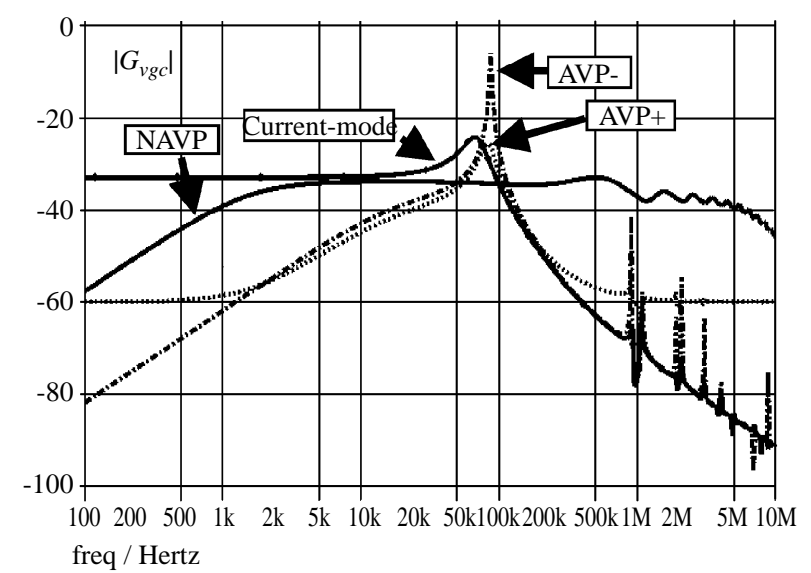

Fig. 8 Closed-loop line regulation (or audio-susceptibility)

concluded that peak current mode control scheme has the advantage in the performance of output impedance and stability margin but is at a disadvantage in line regulation performance.

To preserve the advantages and overcome the disadvantage, a new control scheme was proposed. It's called a current-mode with native AVP scheme (NAVP) shown in the diagram in Fig. 9 (Huang et al., 2007). The diagram is basically a peak current mode control buck converter but with an offset voltage-canceling circuit (the dotted portion). This offset voltage is summed with the error-amplifier output $V_{c}$ to boost $V_{\text {droop }}$, which in turns, cancels out the offset. The offset voltage is obtained by subtraction of $V_{\text {droop }}$ from $V_{c 2}$. Since only DC offset information is needed, a low-pass filter LPF is used to obtain the offset information.

Figure 10 shows the simulated switching waveforms of a current-mode scheme with the offset-canceling circuit. The switching condition of the PWM converter occurs when $V_{c 2}$ meets $V_{\text {droop }}$. Due to the $V_{\text {offset }}$ provided by the offset-canceling circuits, $V_{c}$ is

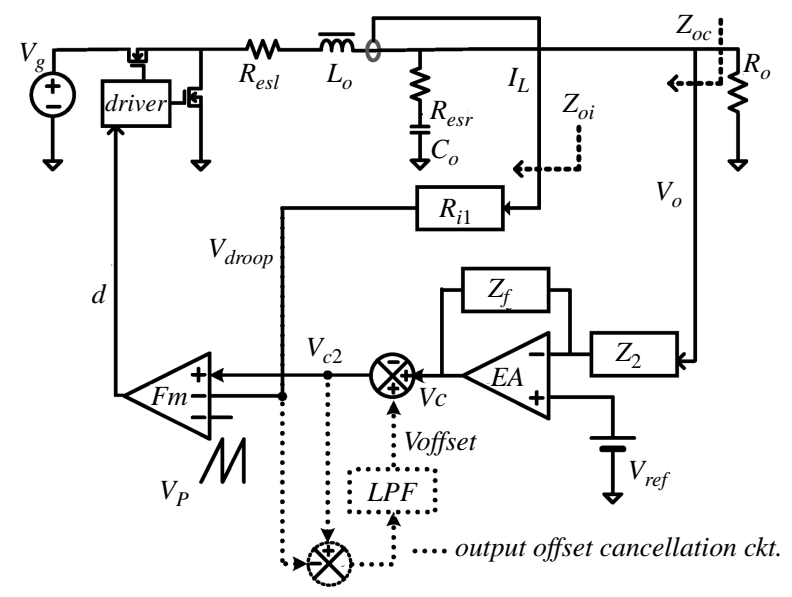

Fig. 9 the circuit diagram of NAVP control

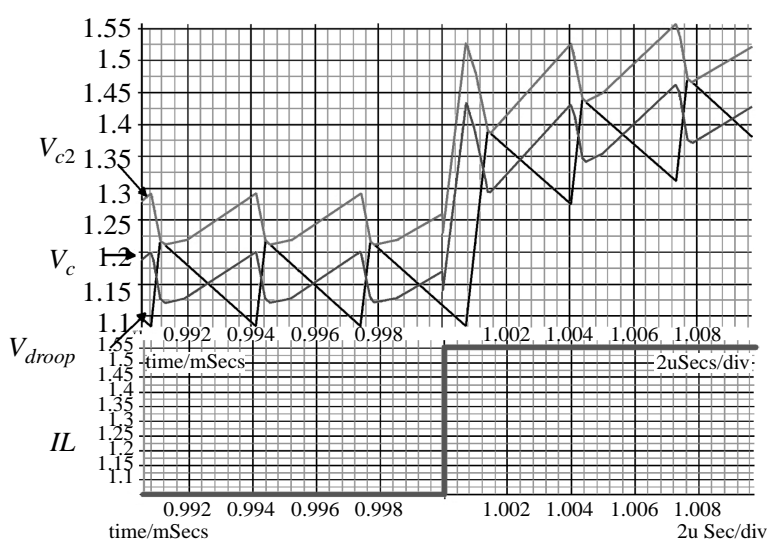

Fig. 10 Simulated waveforms for a step-load condition for a converter with NAVP scheme

set lower by the difference of the averages of $V_{c 2}$ and $V_{\text {droop. }}$. This action eliminates the output voltage offset. If the offset cancelling were removed, then $V_{c 2}$ would set the output voltage which has an offset output voltage in the amount of $\left(V_{2}\right.$-Vdroop $) /\left(2 A V_{o}\right)$ where $A V_{o}$ is the DC gain of the error amplifier. Because of low gain error-amplifier required to achieve current-mode AVP, $A V_{o}$ is normally small (Huang et al., 2007). Therefore the output voltage offset can be significant. As the input voltage increases, the magnitudes of both ripples increase, which causes even larger offsets. This is the reason why the line regulation of such a control is poor. With the NAVP scheme, not only the offset can be eliminated but also the line regulations improved. Fig. 8 shows the NAVP line regulation performance compared with the other three schemes. NAVP therefore, preserves the nice features of current-mode approach such as low $\left|Z_{o c}\right|$ peaking, relatively large stability margin, inherent phase current balancing, and overcomes the disadvantage of relatively poor line regulation in current-mode AVP. 


\section{CONCLUSIONS}

Small-signal loop analysis provides insight into the intricate relationships of the inherent control characteristics of the four schemes mentioned: current-mode, AVP-, AVP+ and NAVP. Because of the constraint of constant output impedance placed on AVP designs, many other control characteristics naturally follow. NAVP scheme seems to compare favorably with the other three schemes in terms of flatness of output impedance curves, line regulations, stability margins, and inherent phase current balancing.

\section{ACKNOWLEDGMENTS}

This work was supported by a grant from the National Science Council of Taiwan (R.O.C.) under Award Number NSC 94-2213-E-002-122 to National Taiwan University. Also the authors would like thank Transim Technology Corporation, U.S.A. for providing the SIMPLIS simulation tool.

\section{REFERENCES}

Huang, J. R., Wang, S., Lee, C., Tseng, E., and Chen, D., 2007, "Native AVP Control Method for Constant Output Impedance of DC Power Converters," Proceedings of IEEE PESC, pp. 2023-2028.
Lee, M., Chen, D., Huang, K., Tseng, E.. and Tai, B., 2006, "Comparisons of Three Control Schemes for Adaptive Voltage Position (AVP) Droop for VRms Applications," Proceedings of IEEE EPEPEMC, pp. 206-211.

Ridley, R. B., Cho, B. H., and Lee, F. C., 1988, "Analysis and Interpretation of Loop Gains of Multiloop-Controlled Switching Regulators," IEEE Transactions on Power Electronics, Vol. 3, No. 4, pp. 489-498.

Ridley, R. B., 1991, "A New Continuous-Time Model for Current-Mode Control," IEEE Transactions on Power Electronics, Vol. 6, No. 2, pp. 271-280.

Yao, K., Meng, Y., Xu, P., and Lee, F. C., 2002a, "Design Considerations for VRM Transient Response Based on the Output Impedance," Proceedings of IEEE APEC, pp. 14-20.

Yao, K., Meng, Y., and Lee, F. C., 2002b, "Control Bandwidth and Transient Response of Buck Converters," Proceedings of IEEE PESC, pp. 137142.

Zhang, M., 2000, "Powering Intel Pentium 4 processors," Intel Technology Symposium, Seattle, WA, USA.

Manuscript Received: June 01, 2007 and Accepted: Aug. 10, 2007 